\title{
Long-term echocardiographic evaluation of asymptomatic patients undergoing minimally invasive valve repair for severe primary mitral regurgitation
}

\author{
Witold Gerber ${ }^{1}$, Krzysztof Sanetra ${ }^{1,2}$, Agnieszka Drzewiecka-Gerber ${ }^{1}$, Justyna Jankowska-Sanetra ${ }^{3}$, \\ Andrzej Bochenek ${ }^{1,4,5}$, Marian Zembala ${ }^{6}$, Marek Cisowski ${ }^{1,5,6}$ \\ 1 1st Department of Cardiac Surgery, American Heart of Poland, Bielsko-Biała, Poland \\ 2 Faculty of Medicine and Health Sciences, Andrzej Frycz Modrzewski Krakow University, Kraków, Poland \\ 3 2nd Department of Cardiology, American Heart of Poland, Bielsko-Biała, Poland \\ 4 Faculty of Medicine, University of Technology, Katowice, Poland \\ 5 Center for Cardiovascular Research and Development, American Heart of Poland, Katowice, Poland \\ 6 Department of Cardiac, Vascular and Endovascular Surgery and Transplantology, Medical University of Silesia in Katowice, Zabrze, Poland
}

\section{KEY WORDS}

cardiac surgery, minimally invasive, mitral valve, remodeling, repair

Correspondence to: Krzysztof Sanetra, MD, 1st Department of Cardiac Surgery, American Heart of Poland, al. Armii Krajowej 101, 43-316 Bielsko-Biała, Poland, phone: +48 334726307 , email: krzyssan@poczta.onet.pl Received: February 10, 2020. Revision accepted: April 7, 2020 Published online: April 8, 2020. Kardiol Pol. 2020; 78 (6): 545-551 doi:10.33963/KP.15287 Copyright by the Author(s), 2020

\section{ABSTRACT}

BACKGROUND Asymptomatic patients with newly diagnosed severe primary mitral regurgitation (MR) may not be candidates for surgery according to clinical guidelines.

AIMS We aimed to determine whether asymptomatic patients with severe primary MR benefit from minimally invasive mitral valve repair.

METHODS This prospective registry study assessed consecutive asymptomatic patients who underwent mitral valve repair using right minithoracotomy. Left ventricular ejection fraction, end-diastolic and end-systolic volumes, end-diastolic and end-systolic diameters, as well as left atrial (LA) area and volume were measured. Major adverse cardiovascular and cerebrovascular events (MACCEs) were assessed at 6 , 12 , and 24 months after surgery.

RESULTS The study included 114 patients, of whom 16 (14\%) were lost to follow-up (except the endpoint of death). No deaths were reported during follow-up. A comparison of median echocardiographic parameters at baseline and 24 months revealed significant reverse remodeling: left ventricular ejection fraction, $68 \%$ vs $60 \%$ ( $P<0.001)$; end-diastolic volume, $165 \mathrm{~cm}^{3}$ vs $107.5 \mathrm{~cm}^{3}(P<0.001)$; end-systolic volume, $51 \mathrm{~cm}^{3}$ vs $43.5 \mathrm{~cm}^{3}(P=0.02)$, end-diastolic diameter, $58 \mathrm{~mm}$ vs $49 \mathrm{~mm}(P<0.001)$; end-systolic diameter, $35 \mathrm{~mm}$ vs $30 \mathrm{~mm}(P<0.001)$; LA area, $26 \mathrm{~cm}^{2}$ vs $18 \mathrm{~cm}^{2}(P<0.001)$; and LA volume, $96 \mathrm{~cm}^{3}$ vs $49.5 \mathrm{~cm}^{3}$ $(P<0.001)$. There were 9 MACCEs $(9.2 \%)$ : 2 reoperations $(2 \%), 1$ hospitalization for heart failure $(1 \%)$, and 6 cases of new-onset atrial fibrillation (6.1\%).

CONCLUSIONS Minimally invasive mitral valve repair is safe and effective in asymptomatic patients with severe primary MR. It should be recommended regardless of ventricular and atrial dimensions.

INTRODUCTION The first mitral valve repair was performed in 1923 by Dr. Elliott Cutler at Peter Bent Brigham Hospital in Boston, Massachusetts. However, it was Alain Carpentier who started the current era of the procedure by outlining the basic pathophysiological classification of mitral valve lesions and providing the guidelines for successful and reproducible mitral valve regurgitation repair. ${ }^{1,2}$

According to the current guidelines, mitral valve repair is the preferred technique when it is expected to be durable (level of evidence Ic). . $^{3,4}$ 


\section{WHAT'S NEW?}

According to the European Society of Cardiology guidelines, many asymptomatic patients with newly diagnosed severe primary mitral regurgitation are not candidates for surgery. However, the combined 5-year incidence of atrial fibrillation, heart failure, and cardiovascular death in nonsurgically managed asymptomatic patients with normal left ventricular function and severe mitral regurgitation was estimated at $42 \%$. In our cohort of asymptomatic patients, no deaths were reported during the 24-month follow-up and the reverse remodeling outcome was highly satisfactory. The minimally invasive access causes less trauma and is more acceptable for patients. Therefore, we strongly recommend the reconstructive surgery in asymptomatic patients with severe regurgitation, regardless of left ventricular and atrial dimensions. The main conclusion of our study is that the left ventricular spherical remodeling rather than end-diastolic diameter should be the most important factor to be considered in the early surgical repair of the mitral valve.

However, numerous asymptomatic patients do not meet the strict criteria for surgery despite having severe mitral regurgitation (MR). Therefore, Heart Teams often face the dilemma of whether to refer a patient for an intervention or whether to recommend watchful waiting. Of note, the 5-year combined incidence of atrial fibrillation, heart failure, and cardiovascular death in nonsurgically managed asymptomatic patients with normal ventricular function and severe MR was estimated at a mean (SD) of $42 \%$ (8\%). ${ }^{5}$ Furthermore, heart failure at 10 years was less frequent after early surgery for severe MR (7\%) than after initial medical treatment (23\%), while survival was estimated at $86 \%$ after the surgery versus $69 \%$ after medical treatment at 10 -year follow-up. ${ }^{6}$

A prospective registry of asymptomatic patients who underwent minimally invasive mitral valve repair revealed a significant reduction of both ventricular and atrial diameters at 6 -month follow-up. ${ }^{7}$ This finding may strongly contribute to a successful clinical outcome, as left atrial and ventricular reverse remodeling seems to play an important role in recovery. Chronic MR is often associated with left atrial (LA) enlargement, which is a well-known predictor of adverse cardiovascular events such as stroke, atrial fibrillation, heart failure, and death. ${ }^{8-10}$ On the other hand, Chipeta et $\mathrm{al}^{11}$ reported a significant reduction in LA volume at 1 week after mitral valve surgery and a further decrease at 6 months, but a slight increase at 12 months after surgery. Therefore, long-term follow-up of asymptomatic patients is indicated. In our study, we aimed to determine whether asymptomatic patients with severe primary MR benefit from minimally invasive mitral valve repair in a long-term follow-up.

METHODS Patients Data for this study were obtained from a prospective institutional registry of minimally invasive mitral valve repair procedures. Consecutive asymptomatic patients who underwent mitral valve repair using right minithoracotomy were selected. Patients were referred for the procedure based on echocardiographic findings and underwent the surgery due to severe primary MR in accordance with the 2012 and 2017 guidelines of the European Society of Cardiology / European Association for Cardio-Thoracic Surgery (ESC/EACTS) (flail leaflet, rupture of the papillary muscle, or large coaptation defect; very large central jet or eccentric jet adhering, swirling, and reaching the posterior wall of the LA; dense or triangular continuous wave signal of the regurgitant jet; large flow convergence zone; vena contracta width $\geq 7$; systolic pulmonary vein flow reversal; E-wave dominant $\geq 1.5 \mathrm{~m} / \mathrm{s}$; the ratio of mitral to aortic time velocity integral $>1.4$; effective regurgitant orifice area $\geq 40 \mathrm{~mm}^{2}$; regurgitation volume $\geq 60 \mathrm{ml} /$ beat; enlargement of the LA/left ventricle [LV]).

Surgery All patients underwent preoperative echocardiographic assessment, and the results were recorded. In patients older than 40 years, additional coronary angiography was performed before admission to a cardiac surgery department. Laboratory and bacteriological tests, as well as any other necessary examinations, were performed prior to hospital admission.

Mitral valve repair was conducted with general anesthesia. Each patient was intubated, and a central venous port was introduced (preferably via the right jugular vein). A transesophageal probe was used for echocardiographic assessment throughout the procedure, and the Foley catheter was passed into the bladder.

Heparin was administered at an adequate dose, calculated according to the patient's body mass. After the activated clotting time of more than 480 seconds was obtained, the cannulation for cardiopulmonary bypass was performed through femoral vessels, using the Seldinger technique. The incision for mitral valve access was made in the fourth intercostal space. Crystalloid cardioplegia (Bretschneider HTK solution or del Nido cardioplegia) was given antegrade into the aorta after cross-clamping the vessel with the Chitwood clamp. After the atriotomy, the final valve assessment and repair were performed. An annuloplasty ring was implanted using single sutures. All other repair procedures were performed using a standard technique. Carbon dioxide was inflated into the operating field until the atriotomy was closed with continuous suture. After the removal of the aortic cross-clamp, transesophageal echocardiography was performed to assess whether the valve function was good (no regurgitation or mild MR). If reintervention was needed, it was performed within the same extracorporeal circulation. Two epicardial electrodes were implanted and the chest tube was introduced into the right pleura. The tube was removed 24 hours after the surgery and follow-up 
radiography was performed. Continuous monitoring of hemodynamic parameters was maintained for 48 hours. Subsequently, the patient was transferred to a cardiac surgery ward for further treatment and rehabilitation. Before discharge, the successful outcome was confirmed on transthoracic echocardiography. On the day of discharge, final electrocardiography and laboratory tests were performed.

Each patient received a vitamin $\mathrm{K}$ antagonist (acenocoumarol or warfarin) at a dose adjusted according to the international normalized ratio

TABLE 1 Baseline demographic and clinical characteristics of the study group $(n=114)$

\begin{tabular}{lll} 
Parameter & Value \\
Age, y, mean (SD) & $48.7(14.9)$ \\
\hline Sex & Male & $79(69.3)$ \\
\cline { 2 - 3 } & Female & $35(30.7)$ \\
\hline Coronary artery disease & $3(2.6)$ \\
\hline Diabetes & $3(2.6)$ \\
\hline Insulin therapy & $1(0.9)$ \\
\hline Arterial hypertension & $41(35.9)$ \\
\hline Asthma / COPD & $1(0.9)$ \\
\hline Hyperlipidemia & $28(24.6)$ \\
\hline Paroxysmal atrial fibrillation & $17(14.9)$ \\
\hline Permanent atrial fibrillation & $5(4.4)$ \\
\hline Obesity & $14(12.3)$ \\
\hline Chronic kidney disease (stage 4 and 5) & $1(0.9)$ \\
\hline BMI, kg/m², mean (SD) & $25.7(4.1)$ \\
\hline
\end{tabular}

Data are presented as number (percentage) of patients unless otherwise indicated.

Abbreviations: BMI, body mass index; COPD, chronic obstructive pulmonary disease

\section{TABLE 2 Surgical procedures in the study group $(n=114)$}

\begin{tabular}{ll} 
Procedure & Value \\
Annuloplasty ring implantation & $114(100)$ \\
\hline Artificial chordae tendineae & $75(65.8)$ \\
\hline P2 resection & $22(19.3)$ \\
\hline P1/P2 cleft closure & $3(2.6)$ \\
\hline P2/P3 cleft closure & $4(3.5)$ \\
\hline Atrial radiofrequency ablation & $14(12.3)$ \\
\hline Left atrial appendage closure & $17(14.9)$ \\
\hline Patent foramen ovale closure & $14(12.3)$ \\
\hline Cross-clamp time, min, mean (SD) & $78.7(16.8)$ \\
\hline Extracorporeal circulation time, min, mean (SD) & $113.6(24.6)$ \\
\hline
\end{tabular}

Data are presented as number (percentage) of patients unless otherwise indicated.

Abbreviations: P1, medial scallop of the posterior leaflet; P2, middle scallop of the posterior leaflet; $\mathrm{P} 3$, lateral scallop of the posterior leaflet
(INR). Low-molecular-weight heparin was administered until the INR exceeded 2. If the patient had no other indications for anticoagulation, vitamin $\mathrm{K}$ antagonist treatment was discontinued 3 months after the procedure. Other medications were administered depending on the patient's clinical status and comorbidities.

Echocardiographic examination All echocardiographic examinations (preoperatively and during follow-up) were performed using a GE Vivid 7 device (GE Healthcare, Chicago, Illinois, United States) by the same echocardiographer. The echocardiographic assessment was scheduled at 6, 12, and 24 months after surgery. The following ventricular and atrial parameters were assessed: LV ejection fraction (LVEF), LV end-diastolic volume, LV end-systolic volume, LV end-diastolic diameter, LV end-systolic diameter (LVESD), LA volume, LA volume index, and LA area.

Other endpoints The assessment for the presence of major adverse cardiac and cerebrovascular events (MACCEs), including death, reoperation, hospitalization due to heart failure, new-onset atrial fibrillation, and stroke, was performed at 6,12, and 24 months. Mortality was assessed using data from the national Polish registry of cardiac surgical procedures (in Polish, Krajowy Rejestr Operacji Kardiochirurgicznych [KROK]), which contains records from the Polish Heart Insurance database, and therefore the follow-up was complete in terms of mortality.

Statistical analysis Data were shown as median (interquartile range [IQR]) or number (percentage). Due to nonnormal distribution of most parameters (D’Agostino-Pearson test), the Mann-Whitney test was used for the analysis of continuous variables. Data were analyzed using the MedCalc software, version 18.5 (MedCalc Software, Ltd., Ostend, Belgium). All tests were 2-tailed, and a $P$ value of less than 0.05 was considered significant.

Ethics All the study procedures were approved by the local Research Ethics Board. Patients provided written informed consent for the procedure and inclusion in the registry.

RESULTS Of the 117 asymptomatic patients who were referred for mitral valve repair, 3 individuals required mitral valve replacement (each received a mechanical valve) due to an unsuccessful repair and were excluded from further analysis in the present report. However, they remained in the database and presented no symptoms at 24-month follow-up.

The baseline demographic and clinical characteristics of the study group are shown in TABLE 1 . The surgery was performed by a single surgeon, 
TABLE 3 Echocardiographic assessment at baseline and at 24-month follow-up

\begin{tabular}{llll} 
Parameter & Baseline & 24 months & P value \\
\hline LVEF, \% & $68(62-73.7)$ & $60(55-65)$ & $<0.001$ \\
\hline LVEDV, cm & $165(128-99.5)$ & $107.5(89-123.5)$ & $<0.001$ \\
\hline LVESV, cm & $51(37-69.7)$ & $43.5(33.5-55)$ & 0.02 \\
\hline LVESD, mm & $35(30-38)$ & $30(27.5-34)$ & $<0.001$ \\
\hline LVEDD, mm & $58(52-63)$ & $49(46.5-51)$ & $<0.001$ \\
\hline LA area, cm ${ }^{2}$ & $26(22.2-30)$ & $18(15.0-24.7)$ & $<0.001$ \\
\hline LA volume, cm ${ }^{3}$ & $96(76.5-122.2)$ & $49.5(38-59)$ & $<0.001$ \\
\hline LAVI, $\mathrm{cm}^{3} / \mathrm{m}^{2}$ & $41.5(30.2-54.4)$ & $25.2(19.4-30.3)$ & $<0.001$ \\
\hline
\end{tabular}

Data are presented as median (interquartile range).

Abbreviations: LA, left atrial; LAVI, left atrial volume index; LVEDD, left ventricular end-diastolic diameter; LVEDV, left ventricular end-diastolic volume; LVEF, left ventricular ejection fraction; LVESD, left ventricular end-systolic diameter; LVESV, left ventricular end-systolic volume who used all necessary means to achieve adequate coaptation and normal valve function (TABLE2). The 16 patients (14\%) who were lost to 24 -month follow-up had similar clinical characteristics and baseline echocardiographic parameters when compared with the remaining cohort.

Echocardiography revealed a significant decrease in LVEF, LV end-diastolic volume, LV end-systolic volume, LV end-diastolic diameter, LVESD, LA volume (both indexed and nonindexed), and LA area (TABLE 3, FIGURES1-3). After indexing, the median (IQR) LA volume was $41.5 \mathrm{~cm}^{3} / \mathrm{m}^{2}\left(30.2-54.4 \mathrm{~cm}^{3}\right.$ / $\left.\mathrm{m}^{2}\right)$ at baseline, $27.8 \mathrm{~cm}^{3} / \mathrm{m}^{2}\left(26.5-29.7 \mathrm{~cm}^{3} / \mathrm{m}^{2}\right)$ at 6 months, $27.7 \mathrm{~cm}^{3} / \mathrm{m}^{2}\left(25.1-29.9 \mathrm{~cm}^{3} / \mathrm{m}^{2}\right)$ at 12 months, and $25.2 \mathrm{~cm}^{3} / \mathrm{m}^{2}\left(19.4-30.3 \mathrm{~cm}^{3} / \mathrm{m}^{2}\right)$ at 24 months, with a significant difference between baseline and 24-month follow-up (TABLE3).

The overall number of MACCEs in the 24-month follow-up was 9 (9.2\%). No deaths were reported during follow-up according to data obtained from the KROK database, and
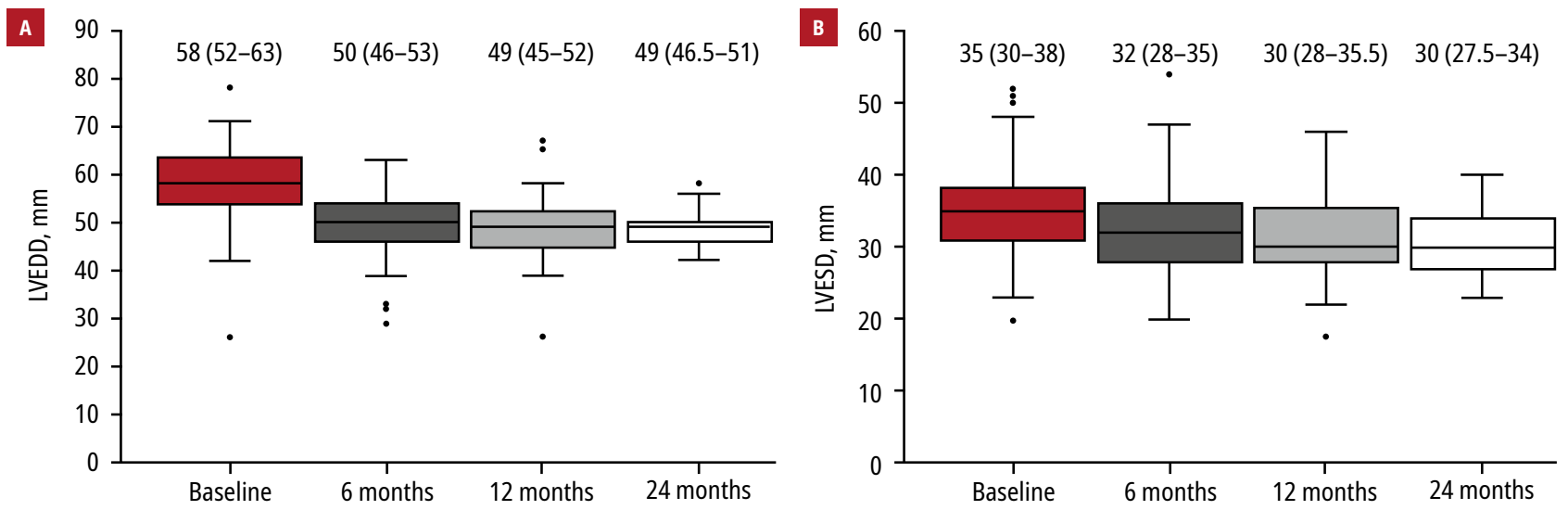

FIGURE 1 Echocardiographic assessment of left ventricular dimensions in long-term follow-up: A - left ventricular end-diastolic diameter (LVEDD); B - left ventricular end-systolic diameter (LVESD). Data are presented as median (interquartile range). The upper and lower borders of the boxes represent the upper and lower quartiles. The middle horizontal line represents the median value. The upper and lower whiskers represent the maximum and minimum values of nonoutliers. The extra dots represent outliers.
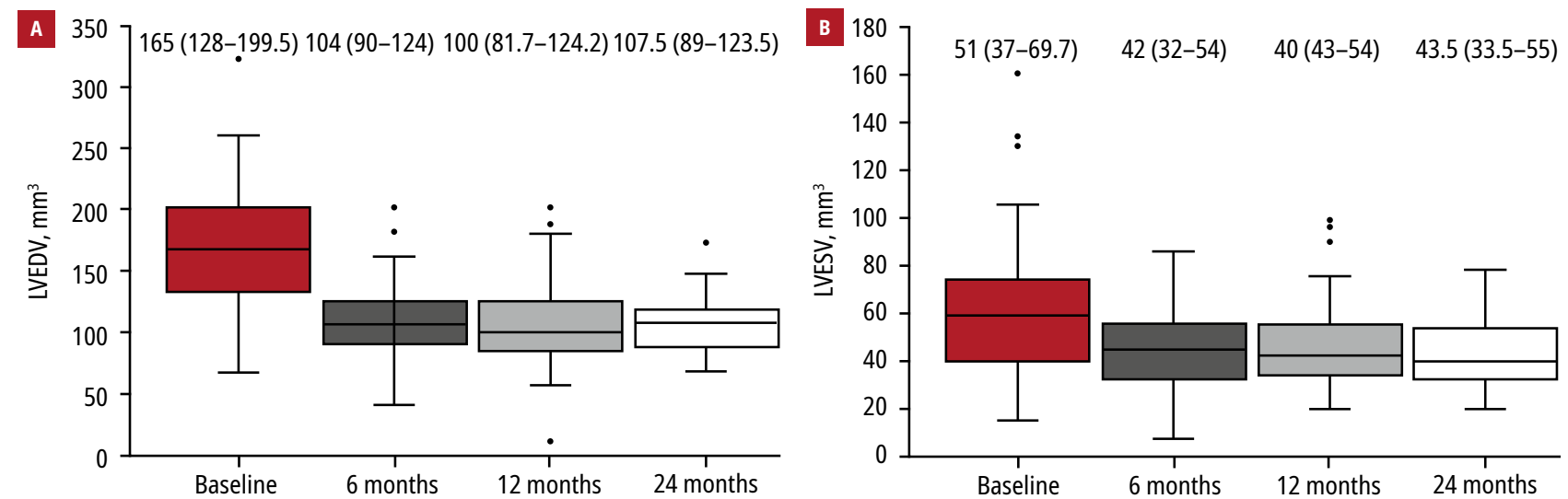

FIGURE 2 Echocardiographic assessment of left ventricular volume in long-term follow-up: A - left ventricular end-diastolic volume (LVEDV); B - left ventricular end-systolic volume (LVESV). Data are presented as median (interquartile range). The upper and lower borders of the boxes represent the upper and lower quartiles. The middle horizontal line represents the median value. The upper and lower whiskers represent the maximum and minimum values of nonoutliers. The extra dots represent outliers. 

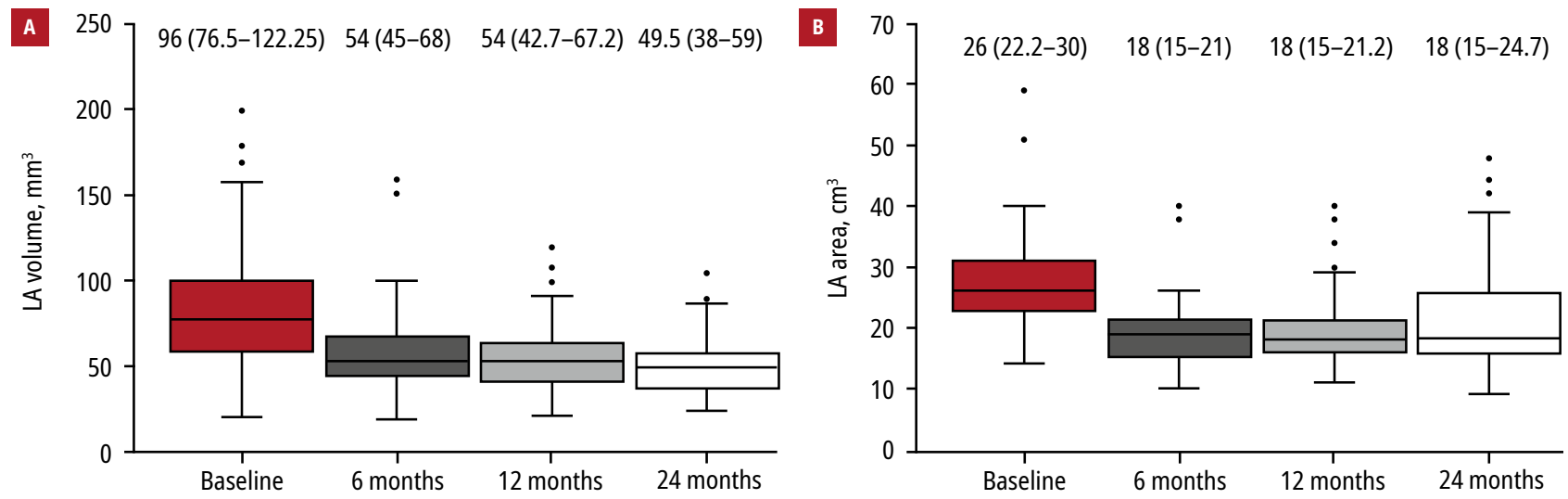

FIGURE 3 Echocardiographic assessment of left atrial (LA) parameters in long-term follow-up: A - LA volume; B - LA area. Data are presented as median (interquartile range). The upper and lower borders of the boxes represent the upper and lower quartiles. The middle horizontal line represents the median value. The upper and lower whiskers represent the maximum and minimum values of nonoutliers. The extra dots represent outliers.

TABLE 4 Major adverse cardiovascular and cerebrovascular events in the 24-month follow-up

\begin{tabular}{ll} 
MACCE & Value \\
Death $^{\mathrm{a}}$ & 0 \\
\hline Reoperation due to valve insufficiency & $2(2)$ \\
\hline Hospitalization due to heart failure & $1(1)$ \\
\hline New-onset atrial fibrillation & $6(6.1)$ \\
\hline Stroke & 0 \\
\hline Total & $9(9.2)$ \\
\hline
\end{tabular}

Data are presented as number (percentage) of patients.

a None of the patients were lost to follow-up regarding the endpoint of death (data from the national registry of cardiac surgical procedures); 16 patients (14\%) were lost to follow-up regarding the other endpoints.

Abbreviations: MACCE, major adverse cardiovascular and cerebrovascular event

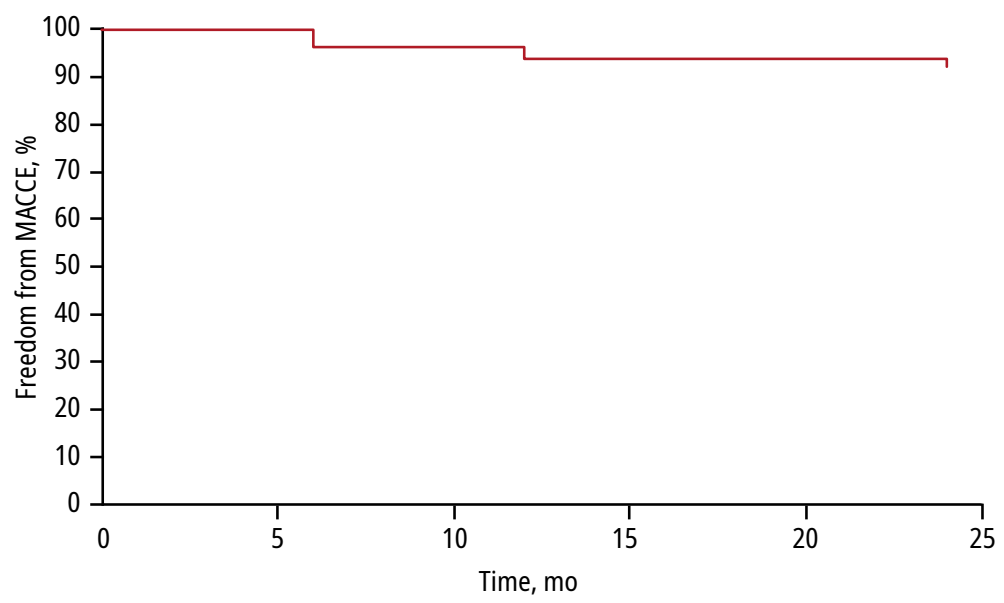

FIGURE 4 Freedom from major adverse cardiovascular and cerebrovascular events (MACCES) during 24-month follow-up: Kaplan-Meier analysis

no patients were lost to follow-up regarding the mortality endpoint. If the follow-up had been extended until the date of the KROK data analysis (median, 48 months), 2 noncardiac deaths would have been reported (one due to malignancy 3 years after the surgery and one due to an infectious disease 4 years after the surgery). Other reported endpoints included new-onset atrial fibrillation, reoperations due to valve insufficiency, and hospitalization due to heart failure. No cases of stroke were reported (TABLE4, FIGURE4).

DISCUSSION This study was primarily designed to verify the 2012 ESC / EACTS criteria for surgical treatment of severe primary mitral valve insufficiency. Asymptomatic patients mostly have preserved LV function, high probability of durable repair, low surgical risk, and LA dilation (IIb indication for surgery).

The issue of indication for surgery seems to be even more complicated in the most recent 2017 ESC / EACTS guidelines for valvular heart disease. ${ }^{3}$ Importantly, the parameters for classifying primary MR as severe remain the same. However, the new guidelines suggest that surgery: 1) is indicated in asymptomatic patients with LV dysfunction (LVESD $\geq 45 \mathrm{~mm}$ and / or LVEF $\leq 60 \%$ ) (level of evidence Ib); 2) should be considered in asymptomatic patients with preserved LV function (LVESD $<45 \mathrm{~mm}$ and LVEF $>60 \%$ ) and atrial fibrillation (level of evidence IIa B); and 3 ) should be considered in asymptomatic patients with preserved LVEF (>60\%) and LVESD of 40 to $44 \mathrm{~mm}$ when a durable repair is likely, surgical risk is low, the repair is performed in heart valve centers, and at least one of the following findings is present: flail leaflet or significant LA dilation (LA volume index $\geq 60 \mathrm{ml} / \mathrm{m}^{2}$ body surface area) in sinus rhythm (level of evidence IIa $C$ ). The modification regarding the LVESD parameter (diameter of $40-44 \mathrm{~mm}$ ), even though the indication advanced from IIb $C$ to IIa $C$, would eliminate even more of our cases from the guideline indication list (the median LVESD was $35 \mathrm{~mm}$ ). The fact that the calculations for patients of small (or 
large) stature may be different does not concern our analysis, as the mean (SD) body mass index in our group was $25.7(4.1) \mathrm{kg} / \mathrm{m}^{2}$.

Although significant reverse remodeling was visible at 6 months, long-term follow-up is necessary, as some authors emphasized that both ventricular and atrial parameters may worsen over time. ${ }^{7,11}$ Marsan et al ${ }^{12}$ reported that $82 \%$ of patients had significant LA reverse remodeling at 6 months after classic mitral valve repair. They also found that the extent of LA remodeling was inversely correlated with age. Our analysis does not support the above data: although a trend towards greater remodeling was noted, the regression model did not show a significant correlation between the patient's age and the degree of remodeling. However, it should be noted that this may not apply to asymptomatic patients, in whom significant remodeling is visible regardless of age.

Numerous other studies also indicated that the size of the LA is significantly reduced after surgery. ${ }^{13-17}$ Importantly, as long as LA dilation persists or progresses, interstitial wall fibrosis and hypertrophy may develop, making reversal remodeling after surgery less likely. ${ }^{18-20}$ An advanced age at the time of surgery also suggests a longer history of MR with less reversible morphological changes of the LA wall. ${ }^{14}$ Some studies strongly indicated that surgery at a younger age may be more beneficial for patients with severe MR, as more significant reverse remodeling can be expected. ${ }^{14,21}$ Machado et al ${ }^{22}$ reported that successful reverse remodeling within the atrium is correlated with normal preoperative LVEF.22

A multicenter study revealed that early surgery in patients with MR due to flail leaflets was associated with a lower incidence of heart failure and lower mortality than medical treatment. However, no difference in the rate of atrial fibrillation, which is usually associated with a large LA, was found. ${ }^{6}$ On the other hand, there are retrospective studies that did not find an association between LA reverse remodeling and reduction in the rates of cardiovascular events, mortality, and atrial fibrillation. ${ }^{23}$

In our study, ventricular dimensions were shown to alter both pre- and postoperatively. Importantly, in most cases, they did not exceed normal values. However, it has been reported that $\mathrm{LV}$ function parameters, such as volume and ejection fraction, may remain compensated despite changes in myocardial properties. ${ }^{24-26}$ Moreover, significant reverse remodeling in all groups suggests that the muscle already changed its geometry due to MR. We have shown that removing severe regurgitation jet and restoring the physiological status results in a significant change in ventricular dimensions. Such a significant reverse remodeling is probably due to an early intervention, as some studies suggest that advanced ventricular hypertrophy and large atrial volume worsen the effect. ${ }^{27}$ In line with this, Suri et $\mathrm{l}^{28}$ reported that recovery in ventricular function was more likely to occur with an early intervention, before the development of ventricular enlargement and dysfunction. A follow-up study of ventricular mass regression following surgery revealed a greater residual LV mass index in patients with reduced preoperative LVEF and secondary tricuspid regurgitation, also suggesting incomplete reverse remodeling and advantages of early surgery. ${ }^{29}$

Importantly, the response to surgery was similar between groups undergoing mitral valve repair and replacement. ${ }^{27}$ However, the repair is favored over replacement because of improved survival, preservation of LV function, and avoidance of prosthetic valve-related complications. ${ }^{3,4}$ The clinical outcomes during and after mitral valve surgery are mostly influenced by the surgical technique and preoperative characteristics of patients, but different etiologies are associated with various types of comorbidities that can indirectly influence the surgical outcome. ${ }^{30}$

Our report clearly supports the idea that patients with severe MR should be considered for an early surgery, before advanced ventricular remodeling occurs. In fact, LV spherical remodeling and not the end-diastolic diameter should be the most important factor to be considered in early surgical repair of the mitral valve.

Our study had several limitations including a single-center design and lack of a control group. Despite this, the presented evidence is strong enough to support the indication for early intervention in asymptomatic patients with severe primary MR. In conclusion, minimally invasive mitral valve repair is a safe and effective modality in asymptomatic patients with severe primary MR. Reverse remodeling of the LV and LA that occurs 6 months after the surgery is maintained during 24-month follow-up and is significant when compared with baseline parameters. Moreover, the intervention should be performed regardless of LV and LA dimensions.

\section{ARTICLE INFORMATION}

\section{CONFLICT OF INTEREST None declared.}

OPEN ACCESS This is an Open Access article distributed under the terms of the Creative Commons Attribution-Non Commercial-No Derivatives $4.0 \mathrm{In}$ ternational License (CC BY-NC-ND 4.0), allowing third parties to download articles and share them with others, provided the original work is properly cited, not changed in any way, distributed under the same license, and used for noncommercial purposes only. For commercial use, please contact the journal office at kardiologiapolska@ptkardio.pl.

HOW TO CITE Gerber W, Sanetra K, Drzewiecka-Gerber A, et al. Long-term echocardiographic evaluation of asymptomatic patients undergoing minimally invasive valve repair for severe primary mitral regurgitation. Kardiol Pol. 2020; 78 : 545-551. doi:10.33963/KP.15287

\section{REFERENCES}

1 Cohn LH, Tchantchaleishvili V, Rajab TK. Evolution of the concept and practice of mitral valve repair. Ann Cardiothorac Surg. 2015; 4: 315-321.

2 Carpentier A. Cardiac valve surgery - the "French correction". J Thorac Cardiovasc Surg. 1983; 86: 323-337. 
3 Joint Task Force on the Management of Valvular Heart Disease of the European Society of Cardiology (ESC); the European Association for Cardio-Thoracic Surgery (EACTS), Vahanian A, et al. Guidelines on the management of valvular heart disease (version 2012). Eur Heart J. 2012; 33: 2451-2496.

4 Baumgartner H, Falk V, Bax J, et al. 2017 ESC/EACTS Guidelines for the management of valvular heart disease. Eur Heart J. 2017; 38: 2739-2791.

5 Grigioni F, Tribouilloy C, Avierinos JF, et al. Outcomes in mitral regurgitation due to flail leaflets. a multicenter European study. JACC Cardiovasc Imaging. 2008; 1: 133-141.

6 Suri RM, Vanoverschelde JL, Grigioni F, et al. Association between early surgical intervention vs watchful waiting and outcomes for mitral regurgitation due to flail mitral valve leaflets. JAMA. 2013; 310: 609-616.

7 Gerber W, Sanetra K, Drzewiecka-Gerber A, et al. Echocardiographic evaluation of the results of minimally invasive repair of asymptomatic mitral regurgitation. Kardiol Pol. 2019; 77: 56-58.

8 Benjamin EJ, D'Agostino RB, Belanger AJ, et al. Left atrial size and the risk of stroke and death. The Framingham Heart Study. Circulation. 1995; 92: 835-841.

9 Messika-Zeitoun D, Bellamy M, Avierinos JF, et al. Left atrial remodelling in mitral regurgitation - methodologic approach, physiological determinants, and outcome implications: a prospective quantitative Doppler-echocardiographic and electron beam computed tomographic study. Eur Heart J. 2007; 28: 1773-1781.

10 Reed D, Abbott RD, Smucker ML, Kaul S. Prediction of outcome after mitral valve replacement in patients with symptomatic chronic mitral regurgitation. The importance of left atrial size. Circulation. 1991; 84: 23-34.

11 Chipeta P, Shim CY, Hong GR, et al. Time course of left atrial reverse remodelling after mitral valve surgery and the impact of left ventricular global longitudinal strain in patients with chronic severe mitral regurgitation. Interact Cardiovasc Thorac Surg. 2016; 23: 876-882.

12 Marsan NA, Maffessanti F, Tamborini G, et al. Left atrial reverse remodeling and functional improvement after mitral valve repair in degenerative mitral regurgitation: a real-time 3-dimensional echocardiography study. Am Heart J. 2011; 161:314-321.

13 Westenberg JIM, van der Geest RJ, Lamb HJ, et al. MRI to evaluate left atrial and left ventricular reverse remodeling after restrictive mitral annuloplasty in dilated cardiomyopathy. Circulation. 2005; 112: 437-442.

14 Antonini-Canterin F, Beladan CC, Popescu BA, et al. Left atrial remodeling early after mitral valve repair for degenerative mitral regurgitation. Heart. 2008, 94: $759-764$

15 Cho DK, Ha JW, Chang BC, et al. Factors determining early left atrial reverse remodeling after mitral valve surgery. Am J Cardiol. 2008; 101: 374-377.

16 Topal AE, Eren MN, Celik Y. Left ventricle and left atrium remodeling after mitral valve replacement in case of mixed mitral valve disease of rheumatic origin J Card Surg. 2010; 25: 367-372.

17 Song $B$, On YK, Jeon ES, et al. Atrioventricular reverse remodeling after valve repair for chronic severe mitral regurgitation: 1 year follow-up. Clin Cardiol. 2010; 33: 630-637.

18 Corin W], Murakami T, Monrad ES, et al. Left ventricular passive diastolic properties in chronic mitral regurgitation. Circulation. 1991; 83: 797-807.

19 Casaclang-Verzosa G, Gersh B], Tsang T. Structural and functional remodeling of the left atrium: clinical and therapeutic implications for atrial fibrillation. J Am Coll Cardiol. 2008; 51: 1-11.

20 Her AY, Choi EY, Shim CY, et al. Prediction of left atrial fibrosis by speckle tracking echocardiography in mitral valve disease: a comparative study with histopathology. Korean Circ J. 2012; 42: 311-318.

21 Cho DK, Ha JW, Chang BC, et al. Factors determining early left atrial reverse remodeling after mitral valve surgery. Am J Cardiol. 2008; 101: 374-377.

22 Machado LR, Meneghelo ZM, Le Bihan DC, et al. Preoperative left ventricular ejection fraction and left atrium reverse remodeling after mitral regurgitation surgery. Cardiovasc Ultrasound. 2014; 12: 45.

23 Lang RM, Bierig M, Devereux RB, et al. Recommendations for chamber quantification: a report from the American Society of Echocardiography's Guidelines and Standards Committee and the Chamber Quantification Writing Group, developed in conjunction with the European Association of Echocardiography, a branch of the European Society of Cardiology. J Am Soc Echocardiogr. 2005; 18: $1440-1463$.

24 Mascle S, Schnell F, Thebault C, et al. Predictive value of global longitudinal strain in a surgical population of organic mitral regurgitation. J Am Soc Echocardiogr. 2012; 25: 766-772

25 Pandis D, Sengupta PP, Castillo JG, et al. Assessment of longitudinal myocardial mechanics in patients with degenerative mitral valve regurgitation predicts postoperative worsening of left ventricular systolic function. J Am Soc Echocardiogr. 2014; 27: 627-638

26 Witkowski TG, Thomas JD, Debonnaire PJ, et al. Global longitudinal strain predicts left ventricular dysfunction after mitral valve repair. Eur Heart J Cardiovasc Imaging. 2013; 14: 69-76.

27 Shafii AE, Gillinov AM, Mihaljevic T, et al. Changes in left ventricular morphology and function after mitral valve surgery. Am J Cardiol. 2012; 110: 403-408.

28 Suri RM, Schaff HV, Dearani JA, et al. Recovery of left ventricular function after surgical correction of mitral regurgitation caused by leaflet prolapse. J Thorac Cardiovasc Surg. 2009; 137: 1071-1076.
29 Stulak JM, Suri RM, Dearani JA, et al. Does early surgical intervention improve left ventricular mass regression after mitral valve repair for leaflet prolapse? J Thorac Cardiovasc Surg. 2011; 141: 122-129.

30 Antunes MJ, Coutinho GF. The conundrum of mitral valve etiology and the association with clinical outcomes. Kardiol Pol. 2019; 77: 505-506. 